\title{
TRANSPORT OF ELECTRON THERMAL ENERGY IN \\ HIGH TEMPERATURE PLASMAS
}

BY

B. COPPI* AND E. MAZZUCATO**

\begin{abstract}
The nature of the anomalous transport of elec:-on thermal energy in existing experiments on magnetically confined toroidal plasma: is discussed and a new form of the relevant electron thermal conductivity, that is consistent with the observed temperature profiles, is presented.
\end{abstract}

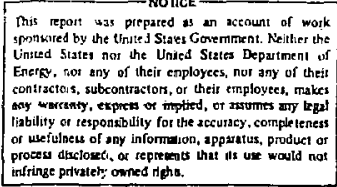

* Massachusetts Institute of Technology, Cambridge, Massachusetts

* Princeton University Plasrna Physics Laboratory, Princeton, New Jersey 08544. This work performed under U.S. Department of Energy Contract No. EY-76-C-02-3073. 
Experiments carried out on the Alcator device since the late Fall of 197/, and later confirmed by results obtained in other confinement experiments, have indicated clearly that the energy replacement time tends to improve as the particle density increases. In fact for most of the experiments carried out so far, the electron population is the main channel for energy loss.

The type of explanation that appears to be most consistent with the more recently observed scalings of temperature and confinement time, is to consider processes that involve primarily the rurrent carrying electron population [1] and magnetic reconnection in the presence of finite electrical resistivity. In fact, we recall that in the collisionless limit, the reconnecting [2] drift-tearing mode that is driven by the current density gradient was found to be stable for a realistic range of value of $\hat{\eta}_{e}=\left(d \ln \mathrm{e}_{\mathrm{e}} / \mathrm{dr}\right) /(\mathrm{d} \ln \mathrm{n} / \mathrm{dr})$ and of its other characteristic parameters.

Then we proceed by dimensional analysis with the intent to reproduce both the temperature and density profiles and the scalings of the electron energy confinement time in experiments carried out on the Alcator, the PLT, and the Frascati Torus devices. The indication of these experiments is that the energy replacement time is relatively insensitive to the value of the toroidal field, while it degrades when $I /\left(\mathrm{nT}_{\epsilon}\right)$ is increaseri ( $\mathrm{I}$ is the toroidal plasma current), in a given configuration at constant values of plasma radius.

It is convenient to represent the experimental results in terms of an effective electron energy replacement time $T_{E e}$ that is defined as

$$
\tau_{E e} V I=\frac{3}{2}<n T_{e}>2 \pi^{2} a^{2} R
$$

where $V$ is the resistive toroidal voltage applied on the plasma. In fig. 1 the values of Ee which were obtained by the FT, PLT and Alcator $[3,4,5]$ experiments are shown as a function of the parameter $\tau^{*}=\kappa\left(k n T_{e}>/ B_{p}\right) a^{2}(a / R)$, where $B_{p}$ is the poloidal magnetic field at the limiter radius and $\alpha$ is a nearly constant quantity. We have also included, in fig. 1 , typical results from the T-10 and DITE devices $[6,7]$. 
These data were collected in the following range of plasma parameters:

$9 \mathrm{~cm} \leq \mathrm{a} \leq 40 \mathrm{~cm}, 28 \mathrm{kG} \leq \mathrm{B}_{\mathrm{T}} \leq 80 \mathrm{kG}, 4 \times 10^{13} \mathrm{~cm}^{-3} \leq \mathrm{n}_{\mathrm{eo}} \geq$

$1 \times 10^{15} \mathrm{~cm}^{-3}, 400 \mathrm{eV} \leq \mathrm{T}_{\mathrm{eo}} \leq 1200 \mathrm{eV}, 120 \mathrm{kA} \leq 1 \leq 550 \mathrm{kA}$.

Moreover, in order to minimize the contribution of impurity losses to the electron energy balance, we have analyzed only plasma regimes with $z_{\text {eff }} \simeq 1$.

Now we refer to weakly collisional regimes and we associate magnetic reconnection processes, that affect the distribution of the poloidal field, with the effects of finite electrical resistivity. We proceed by dimensional analysis and consider characteristic scale distances that affect magnetic reconnection processes and current carrying, circulating, electrons

$d=\frac{c}{\omega_{p e}}$ and $(\Delta r)_{e}=\rho_{\theta e} \frac{r}{R}$,

These are, respectively, the inertial skin depth and the average radial width of circulating electron orbits on the magnetic surface of minor radius $r$. Here $\omega_{p e}$ is the Langnuir frequency, $\rho_{\theta \mathrm{e}}=v_{\text {the }} / \boldsymbol{\Omega}_{\theta \mathrm{e}}, \boldsymbol{\Omega}_{\theta \mathrm{e}}=\mathrm{eB}_{\theta} /\left(\mathrm{m}_{\mathrm{e}} \mathrm{c}\right)$, and $B_{\theta}$ is the poloidal magnetic field component. Recalling the theory of collisionless drift-tearing modes [2], we introduce the distance

$d_{m}=\frac{d^{2}}{(\Delta r)_{e}} \propto \frac{B_{\theta}}{n_{e} T_{e}^{1 / 2}} \frac{R}{r}$

and describe the electron thermal diffusion by the coefficient

$D_{\text {eff }} \propto d_{m} v_{m}$

Here $v_{m}$ is the diffusion velocity that we express in terms of a characteristic fractional power of the resistive diz Eusion coefficient $D_{\eta}=\eta_{1} c^{2} /(4 \pi) \propto \mathrm{z}_{\text {eff }} / \mathrm{T}_{\mathrm{e}}^{3 / 2}$, where $\mathrm{z}_{\text {eff }}$ is the effective ion charge number. Then we have 
$v_{m} \propto\left(D_{\eta} \omega_{p i} v_{\text {the }}^{1 / 2}\right)^{2 / 5}$

where $\omega_{p i}^{2}=4 \pi n e^{2} z_{e f f} / m_{i}$, and for $n^{*} \equiv n z_{\text {eff }} / A_{i}, A_{i}$ being the ion mass number and $\epsilon_{D}$ a small numerical coefficient,

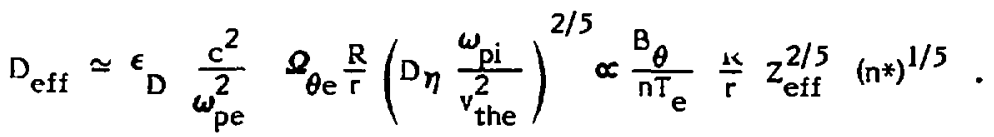

If we rewrite the total thermal energy balance for the case where only ohmic heating is present and the electrons provide the main channel for energy loss we find the following scaling for the average temperature

$\left\langle\tau_{e}\right\rangle \propto\left(\left\langle\frac{\eta_{I l}}{\eta_{c l}}>B_{p} \frac{a}{R}\right)^{2 / 3}\left(\frac{\left\langle z_{\text {eff }}\right\rangle}{\left\langle n^{*}\right\rangle^{1 / 3}}\right)^{2 / 5} \propto\left(\frac{\mathrm{I}}{R}\right)^{2 / 3}\left(\frac{\left\langle z_{\text {eff }}\right\rangle}{\left\langle n^{*}\right\rangle}\right)^{1 / 3}\right)^{2 / 5}\left\langle\frac{\eta_{\text {II }}}{\eta_{c l}}\right\rangle^{2 / 3}$,

where $B_{P}=B_{\theta} \quad(r=a) \propto I / a$, and $\eta_{11} / \eta_{C \ell}$ takes into account the effects of trapped electrons on the longitudinal resistivity.

We notice that the scaling (6) has been most clearly indicated by the series of experiments carried out in the Frascati Torus [3]. According to eq. (5) the scaling for the energy replacement time would become

$$
\tau_{E} \propto \frac{a^{3}}{R} \frac{\left\langle n T_{e}\right\rangle}{B_{p}\left(\left\langle z_{e f f}\right\rangle^{2}\left\langle n^{*}\right\rangle\right)^{1 / 5}},
$$

and in the case of prevalent ohmic heating,

$$
T_{E} \propto<n>a^{2} \frac{1}{\left(B_{p} n^{*}\right)^{1 / 3}}\left(\frac{a}{R}\right)^{5 / 3}<\frac{\eta_{11}}{\eta_{c l}}{ }^{2 / 3}
$$

We notice that, apart from the almost constant factor $\left\langle n^{*}\right\rangle^{1 / 5}$, this scaling agrees with that of fig. 1 when $\eta_{\|} \propto \eta_{\mathrm{cl}}$. Then we consider the local electron thermal energy balance equation in the form 
$E_{\| I} J_{\|}=-\frac{1}{r} \frac{d}{d r}\left(r n D_{\text {eff }} \frac{d}{d r} T_{e}\right)$

where $J_{\|}=(c / 4 \pi)(1 / r) d\left(B_{\theta} r\right) / d r$ and $E_{\|}$is independent of $r$. We notice that eq. ( 9$)$ admits the "diffusion" profile

$T_{e}=T_{e}^{o} \exp \left[-r^{2} /\left(\alpha_{D} a^{2}\right)\right]$

for

$D_{\text {eff. }}=\alpha_{D} \frac{c}{8 \pi} \vee \frac{B_{\theta}}{n T_{e}} \frac{R}{r} \frac{a^{2}}{2 \pi R^{2}}$

where $\boldsymbol{\alpha}_{D}=$ const. Therefore the form (5) is equivalent to state that, in the "diffusion" profile that it generates, $\alpha_{D}$ is not exactly constant but is a weak function of $n$ and $z_{\text {eff }}$. In particular, given the scaling (5), we have

$\alpha_{D} \approx \alpha_{D o} \quad\left(n * /<n^{*}>\right)^{1 / 5} \quad\left(z_{\text {eff }} /<z_{\text {eff }}>\right)^{2 / 5}$,

where $\alpha_{D o}$ is a constant.

Notice that the profile $(10)$ is very similar to those that are obtained experimentally $[3,4]$. If we combine eq. (11) with (5) we obtain the following scaling for $V$

$v \propto\left\langle z_{e f f}\right\rangle^{2 / 5}\left(\frac{R}{a}\right)^{2}\left\langle n^{*}>^{1 / 5}\left(\frac{<T_{e}>}{T_{e}^{o}}\right)^{-1}\right.$.

It is possible that the special form of the diffusion coefficient that is found to interpret the experiments is the direct consequence of a continuing relaxation process. This can be a result of the condition that the established current density and temperature profiles are consistent with the constraints of toroidal macroscopic equilibrium for the considered confinement configuration.

The total energy replacement time $\tau_{E}$ is related to the electron and ion energy confinement times, $\tau_{e}$ and $\tau_{i}$ by,

$\frac{1}{\tau_{E}}\left\langle n\left(T_{e}+T_{i}\right)\right\rangle=\frac{1}{T_{e}}\left\langle n T_{e}\right\rangle+\frac{1}{\tau_{i}}\left\langle n T_{i}\right\rangle$ 
and if we refer to the respective rates of energy loss $\nu_{E L}, \nu_{\text {eL }}$ and $\nu_{\text {iL }}$ we have $\nu_{\mathrm{EL}}=\left\{\nu_{\mathrm{eI}}+\nu_{\mathrm{iL}} \frac{\left\langle\mathrm{nT}_{\mathrm{i}}\right\rangle}{\left\langle\mathrm{nT} \mathrm{T}^{\rangle}\right.}\right\} \frac{\left\langle\mathrm{nT} \mathrm{e}_{\mathrm{e}}\right.}{\left\langle n\left(\mathrm{~T}_{\mathrm{e}}+\mathrm{T}_{\mathrm{i}}\right)^{\rangle}\right.}$.

If we consider relatively high density regimes where $T_{i} \simeq T_{e}$, the contribution of $\nu_{i L}$ due to (discrete) collisions can become comparable with that of $\nu_{\mathrm{eL}}$. Thus if we take $\nu_{\mathrm{eL}} \approx \mathrm{D}_{\mathrm{eff}} / \mathrm{a}^{2}$, and recall that the collisional contribution of $\nu_{\mathrm{iL}}$ scales as $\mathrm{nq}_{\mathrm{a}}^{2}\left(\mathrm{~B}^{2} \mathrm{~T}_{\mathrm{i}}^{1 / 2}\right)$, the type of dependence of $\nu_{E L}$ on $n$ that is obtained is illustrated in fig. 2. This was in fact proposed [8] also in 1975 and by now a number of experiments appear to confirm it.

The observed temperature profiles correspond to values of $\epsilon_{D}$ around $0.5 \times 10^{-2}$. In addition, at the outer edge of the plasma column, where $n$ and $T_{e}$ tend to vanish, the aropted expression for $D_{\text {eff }}$ is taken to have $D_{B} \equiv c T_{e} /(e B)$ as an upper bound [9]. Likewise, if there is a region at the center of the plasma column where $q(r) \leq 1, D_{\text {eff }}$ is taken to be equal to $D_{B}$ in order to simulate enhancement of transport due to $m^{\circ}=1$ modes if the degree of collisionality is sufficiently high for their excitation [2].

Diffusion coefficients based on the existence of a turbulent pattern of reconnected magnetic surfaces have been proposed by Ohkawa [10], Kadomtsev [11], and Rosenbluth [12]. The relevant expressions are typical of the form $\mathrm{D}_{\text {eff }} \simeq\left(\mathrm{c} / \omega_{\mathrm{pe}}\right)^{2} v_{\text {the }} /(\mathrm{gR}) \propto \mathrm{T}_{\mathrm{e}}{ }^{1 / 2} \mathrm{~B} /\left(\mathrm{rB}_{\theta} \mathrm{n}\right)$. However if we adopt this type of diffusion coefficient in a numerical transport code we would see that it fails to reproduce the observed temperature profiles on account of the positive coefficient of the electron temperature.

On the other hand the diffusion coefficient proposed in ref. [1], $\mathrm{D}_{\text {eff }} \propto \mathrm{J}_{\| 1} /\left(\mathrm{nRT}_{\mathrm{e}}{ }^{1 / 2}\right)$ had appropriate type of exponents of the quantities involved in it in order to reproduce the observed profiles, but it had to be complemerited with another different coefficient [9] in order to increase the rate of energy loss near the outer edge of the plasma column. 
Finally we notice that if we assume, as is frequently done, that the achievable plasma density scales as $B / R$, we have a consequence, $n \tau_{e} \propto B^{5 / 3}(a / R)^{10 / 3} q_{a}{ }^{1 / 3}$. Thus, an ohmically heated device with $a=40 \mathrm{~cm}, \mathrm{R}=85 \mathrm{~cm}, \mathrm{~B}_{\mathrm{T}}=120 \mathrm{kG}$, should achieve $\langle n\rangle \approx 5 \times 10^{14} \mathrm{~cm}^{-3},\left\langle T_{\mathrm{e}}\right\rangle \approx\left\langle\mathrm{T}_{\mathrm{i}}\right\rangle=2 \mathrm{keV}$ and $\left.n_{\mathrm{o}} \tau_{\mathrm{E}}\right\rangle 1 \times 10^{14} \mathrm{~cm}^{-3} \mathrm{sec}$ for $I \simeq 4.2$ MA corresponding to $q_{\mathrm{a}}=2.7$.

This work was sponsored in part by the U.S. Department of Energy, Contract No. EY-76-6-02-3073. We wish to thank T. Antonsen, D. Duchs, G. Rostagni, and A. Taroni for their comments and contribution. 


\section{REFERENCES}

[1] B. Cuppi, G. Lampis and F. Pegoraro, Phys. Letters, 59A, 118 (1976).

[2] B. Coppi, J. Mark, 2. Sugiyama and G. Bertin, in RLE Report PRR-78/21 (Massachusetts Institute of Technology, Cambridge, Mass.: 1978), to be published in Annl. Phys.

[3] U. Ascoli-Bartoli, G. Bardotti, R. Bartiromo, G. Battaglia, M. Bruscati, P. Buratti, G. Cima, A. De Angelis, F. De Marco, L. Enriques, M. Gasparotto, M. Grolli, M. Haegi, R. Marconero, M. Martone, G. Mazzitelli, E. Mazzucato, L. Pieroni, G.B. Righetti, M. Samuelli, S.E. Segre, C. Strangio and A. Tuccillo, in Plasma Physics and Controlled Nuclear Fusion Research (Publ. IAEA, Vienna, 1978).

[4] K. Bol, V. Arunasalam, M. Bitter, D. Boyd, K. Brau, N. Bretz, J. Bussac, S. Cohen, P. Colestock, S. Davis, D. Dimock, F. Dylla, D. Eames, P. Efthimion, H. Eubank, R. Goldston, R. Hawryluk, K. Hill, E. Hinnov, J. Hosea, H. Hsuan, F. Jobes, D. Johnson, E. Mazzucato, S. Medley, W. Stodiek, J. Strachan, S. Suckewer, G. Tait, M. Ulrickson and S. von Goeler, in Plasma Physics and Controlled Nuclear Fusion Research (Publ. IAEA, Vienna, 1978).

[5] M. Gaudreau, A. Gondhalekar, M.H. Hughes, D. Overskei, D.S. Pappas, R.R. Parker, S.M. Wolfe, E. Apgar, H.I. Helava, I.H. Hutchinson, E.S. Marmar and K. Molvig, Phys. Rev. Lett. 39, 1266 (1977).

[6] A.A. Bagdasarov, A.B. Berlizov, N.L. Vasin, A.N. Vertiporokh, V.P. Vinogradov, E.P. Gorbunov, Yu.N. Dnestroskij, Yu.N. Esipchuk, V.S. Zaveryaev, A.B. Izvozchikov, A.Ya. Kislov, S.Yu. Lykyanov, S.E. Lysenko, Yu.S. Maximov, G.E. Notkin, G.V. Pereverzev, K.A. Razumova, V.S. Strelkov and D.A. Shcheglov, in Plasma Physics and Controlled Nuclear Fusion Research (Publ. IAEA, Vienna, 1978). 
[7] K.B. Axon, G.A. Baxter, J. Burt, W.H.M. Clark, G.M. McCracken, S.J. Fielding, R.D. Gill, D.H.J. Goodall, M. Hobby, J. Hugill, J.W.M. Paul, B.A. Powell, R. Prentice, G.W. Reid, P.E. Stott, D.D.R. Summers and A.J. Wootton, in Plasma Physics and Controlled Nuclear Fusion Research (Publ. JAEA, Vienna, 1978).

[8] B. Coppi, RLE Report PRR-75/27 (Massachusetts Institute of Technology, Cambridge, Mass., 1975).

[9] B. Coppi and A. Taroni, In Proceedings of the 1977 Varenna Symposium on Magnetically Confined Plasmas (Publ. Pergamon Press and Euratom, Brussells, 1978).

[10] J. Ohkawa, Report GA-414433, General Atomic, San Diego, Cal. (1977).

[11] B.B. Kadomtsev, in Plasma Physics and Controlled Nuclear Fusion Research (Publ. IAEA, Vienna, 1978).

[12] M.N. Rosenbluth, Bull. Am. Phys. Soc. 23, 761 (1978). 


\section{FIGURE CAPTIONS}

Fig. 1. Scaling of the effective electron energy replacement time $\tau_{\text {Ee }}[$ msec $]$, as a function of the parameter

$$
T^{*} \equiv 0.8<\mathrm{n}_{\mathrm{e}}\left[10^{14} \mathrm{~cm}^{-3}\right] \cdot \mathrm{T}_{\mathrm{e}}[\mathrm{keV}]>\mathrm{a}^{2}\left[\mathrm{~cm}^{2}\right](\mathrm{a} / \mathrm{R}) / \mathrm{B}_{\mathrm{p}}[\mathrm{kG}]
$$

Fig. 2. Scaling of the energy loss rate $\nu_{1}$ versus the particle density $n$ when the thermal conductivity of electrons is anomalous and that of ions is collisional, for increasing values of the magnetic field $B$ and constant values of the safety factor $q_{a}$ 
$-11-$

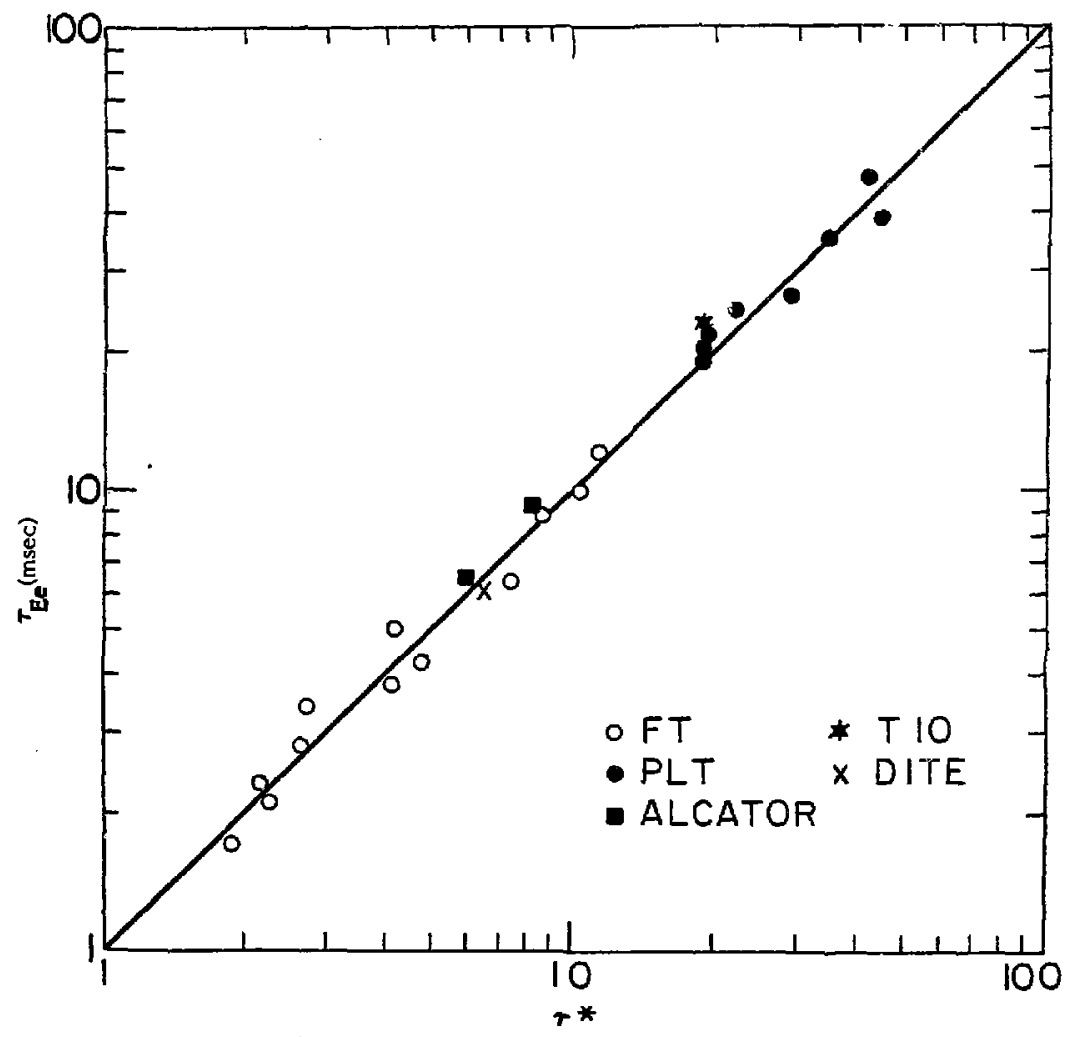

Figure 1 
$-12$.

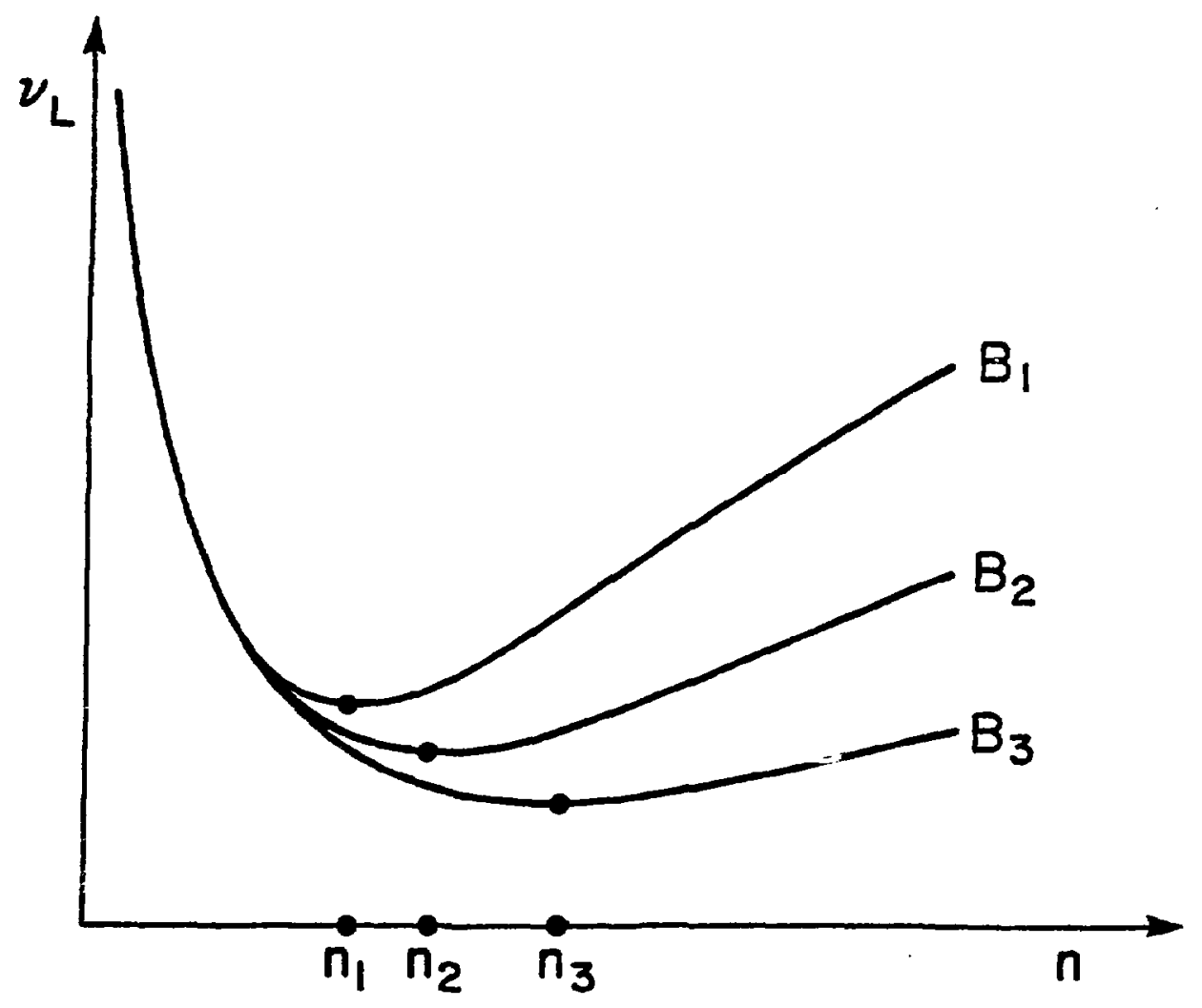

Figure 2 